\title{
Surgical management and outcome of rectal carcinoids in a university hospital
}

\author{
Rockson Wei", Oswens SH Lo and Wai Lun Law
}

\begin{abstract}
Background: Rectal carcinoids are an uncommon entity comprising only 1\%-2\% of all rectal tumors. Rectal carcinoids are frequently diagnosed during colonoscopy, but management after polypectomy is still controversial. The aims of this study were to review the surgical procedures for rectal carcinoids and to compare the outcomes of patients after different treatment modalities in a university hospital in Hong Kong.

Methods: All rectal carcinoids diagnosed between January 2003 and September 2012 were reviewed retrospectively, including clinicopathological characteristics, their management, and surgical outcomes.

Results: There were 54 patients with a median age of 60 years, and 32 were males (59.3\%). All patients underwent colonoscopy, and the most had rectal bleeding (53.7\%). Two patients were diagnosed incidentally in the surgical specimens of rectal tissues. Eighteen patients were diagnosed to have rectal carcinoids after snaring polypectomy, and no further intervention was required. Twenty-five patients had local resection either by means of transanal resection or transanal endoscopic operation. Radical resection was performed in seven patients in which one had T3N1 disease and the others did not have any lymph node metastasis.

In the median follow-up of 30 months (10-108 months), there was no recurrence in the "incidental" or post-polypectomy group. However, two patients with transanal resection and two patients with radical resection developed hepatic metastases after 13-24 months post-treatment. The 5-year overall survival was 100\% in patients having snaring polypectomy only, $83 \%$ for those with local resection, and $63 \%$ in patients who underwent radical surgery $(p=0.04)$.

Conclusions: Our data suggested that that local resection was an effective treatment for small rectal carcinoids and generally brought about good oncological and surgical outcomes. For larger tumors, radical resection seemed to provide acceptable oncological outcomes. Regular surveillance with colonoscopy and endorectal ultrasound is highly recommended for high-risk patients for long-term management. By sharing our experience, we hope to provide more evidence on the management on rectal carcinoids which, together with evidence from further studies, may guide us in the long-term management of these patients in the future.
\end{abstract}

\section{Background}

Carcinoid tumors, a type of neuroendocrine tumors, comprise of a heterogeneous group of neoplasms arising from enterochromaffin cells. These relatively uncommon tumors have variable histological patterns and biologic behaviors $[1,2]$. Rectum is the third most common site of occurrence for carcinoids, with up to $13.7 \%$ of all carcinoids found at that site. On the other hand, carcinoids only comprise of $1 \%-2 \%$ of all rectal tumors [3,4]. Both the overall incidence of carcinoid tumors as well as the proportion of rectal carcinoids in all rectal tumors has

\footnotetext{
* Correspondence: rockson24@yahoo.com

Division of Colorectal Surgery, Department of Surgery, The University of Hong Kong, Queen Mary Hospital, Pok Fu Lam, Hong Kong
}

been increasing drastically in recent years [3,4]. The ageing population, increased rate of screening with colonoscopy, and the increased awareness of the clinicians probably account for this change in incidence [3]. Concerning treatment, studies of different populations showed that sizes of rectal carcinoid lesions are usually small [2-4]. While larger lesions require major rectal resection, most can be treated with local excision either using endoscopic means or via transanal route with good surgical outcomes [5,6]. However, there is still no consensus on the treatment strategy and surveillance of rectal cancer in general yet $[5,7,8]$.

The aims of this study were to review the surgical procedures for rectal carcinoids and to compare the outcomes of 
patients after different treatment modalities in a university hospital in Hong Kong.

\section{Methods}

\section{Patient inclusion}

All patients who had treatment of rectal carcinoids at Queen Mary Hospital between January 2003 and September 2012 were included in this study. All tumors were located within $15 \mathrm{~cm}$ from anal verge. Data on patients' demographics, clinical presentations, treatment modalities, recurrence, and survival were retrospectively reviewed and analyzed. The tumor size was defined by the largest diameter of the tumor. Other histopathological characteristics, such as resection margins, mitotic rate, and immunostaining features, were searched for and analyzed if available.

\section{Treatment methods}

Endoscopic treatment such as snaring polypectomy or endoscopic mucosal resection resulting in adequately clear margins was considered as curative treatment for small rectal carcinoids $(<10 \mathrm{~mm})$. For larger rectal carcinoids $(>10 \mathrm{~mm}$ ) or those with positive margin after polypectomy, repeated resection was performed. Local excisional procedures, either transanal resection or transanal endoscopic operation (TEO), would be performed. Radical rectal resection (such as low anterior resection) was performed in patients with potentially aggressive carcinoids or concomitant rectal adenocarcinoma.

All patients were followed up in outpatient clinic with digital examinations performed at 2 to 4 weeks after operation, and then at every 2-3-month intervals in the first and second year. Follow-up appointments would then be spaced out to yearly afterwards. We offer lifelong follow-up assessment. Surveillance colonoscopy would be arranged to look for any local recurrence or metachronous colorectal malignancy in a 3-yearly interval. CT scan was used only in 3- to 5-year intervals to look for distant metastasis in patients who presented with poor prognostic features.

\section{Statistical methods}

Data were analyzed with PASW version 18. Continuous variables were analyzed with $t$-test, whereas categorical variables were compared with chi-square test. Kaplan Meier survival curve was used for survival analysis, and comparison was made with log-rank test. $p$ values of less than 0.05 were considered significant.

\section{Results}

During the study period, there were 54 patients diagnosed to have rectal carcinoids and all were of Chinese ethnicity. Thirty-two patients were males (59.3\%), and the median age was 61 among them all (range: 29 to 88 years). The most common presenting symptom was rectal bleeding (53.7\%). Only one patient had symptoms of carcinoid syndrome with spurious diarrhea and flushing. In large carcinoid tumors, $(>10 \mathrm{~mm})$, statistically significant association was shown with symptoms of anal pain or discomfort $(p=0.024)$ but not with bleeding $(p=0.349)$ (Table 1).

In most patients, the diagnosis of carcinoid was made by endoscopic examination and biopsy except for two patients who were diagnosed to have rectal carcinoids after surgical procedures for seemingly unrelated diseases; one after hemorrhoidectomy and the other after the transrectal prostate biopsy, with the specimens showing the presence of rectal carcinoid.

Table 1 Data on patients' characteristics

\begin{tabular}{|c|c|c|c|c|}
\hline & Overall & Size $<10 \mathrm{~mm}$ & Size $<10 \mathrm{~mm}$ & $p$ values \\
\hline Age (median) & 60 & 57 & 65 & 0.716 \\
\hline Male:female & $32: 22$ & $26: 16$ & $6: 6$ & 0.382 \\
\hline \multicolumn{5}{|l|}{ Presenting symptoms } \\
\hline Bleeding & $28(51.9 \%)$ & $20(37.0 \%)$ & $8(14.8 \%)$ & 0.349 \\
\hline Tenesmus & $6(11.1 \%)$ & $4(7.4 \%)$ & $2(3.7 \%)$ & 0.547 \\
\hline Anal/rectal mass & $7(13.0 \%)$ & $3(5.6 \%)$ & $4(7.4 \%)$ & 0.024 \\
\hline Anal pain/discomfort & $7(13 \%)$ & $3(5.6 \%)$ & $4(7.4 \%)$ & 0.024 \\
\hline Carcinoid syndrome & $1(1.9 \%)$ & $0(0 \%)$ & $1(1.9 \%)$ & 0.069 \\
\hline \multicolumn{5}{|l|}{ Treatment modalities } \\
\hline Snaring polypectomy & $18(33.3 \%)$ & $0(0 \%)$ & $18(33.3 \%)$ & \\
\hline Transanal resection & $10(18.5 \%)$ & $4(7.4 \%)$ & $6(11.1 \%)$ & \\
\hline TEO & $15(27.5 \%)$ & $3(5.6 \%)$ & $12(22.2 \%)$ & \\
\hline Radical resection & 7 (13.0\%) & $3(5.6 \%)$ & $4(7.4 \%)$ & \\
\hline No treatment & $4(7.4)$ & $2(3.7 \%)$ & $2(3.7 \%)$ & \\
\hline
\end{tabular}


Most gastrointestinal carcinoids can be recognized during routine microscopic examination, but immunohistochemical staining is a useful aid. Chromogranin A, a glycoprotein stored in secretory granules of neuroendocrine cells, was positive in $75.6 \%$ of the patients. Synaptophysin, a neuron-specific enolase test, was found positive in $97.9 \%$ of the patients.

The median size of the rectal carcinoids was $5 \mathrm{~mm}$, and it ranged from 1 to $60 \mathrm{~mm}$. Seventeen patients with small tumors were treated by snaring polypectomy alone, and one patient had endoscopic mucosal resection. The median size of the tumor in this group of patients (group 1) was $4.5 \mathrm{~mm}$ (range: 1 to $8 \mathrm{~mm}$ ). The indications for further intervention after snaring polypectomy included incomplete endoscopic removal or positive resection margins (Table 2), and the 25 patients belonging to this group (group 2) underwent either transanal resection $(18.5 \%)$ or TEO (27.8\%). The median tumor size in this group of patients was larger than that in the previous group, being $6 \mathrm{~mm}$ (range: 1.5 to $25 \mathrm{~mm}$ ). The third group (group 3) consisting of seven patients (13.0\%) who had radical resection for tumors of larger size, concomitant rectal adenocarcinoma, or margin involvement (Table 2). Of these seven patients, one had T3N1 disease and the other had T2 or less without lymph node metastasis. Two other patients had metastatic disease on presentation and therefore did not undergo any surgical treatment.

During the median follow-up of 30 months (10 to 108 months), no local or systemic disease recurrence was detected in the polypectomy alone group (group 1). In the local resection group (group 2), three patients had disease recurrence after transanal resection. None of the patients who underwent TEO had disease recurrence. One patient had local recurrence after 24 months and was disease free for 35 months after another transanal resection. The other two patients developed and subsequently died of systemic recurrence, one at 5 months and the other at 13 months after initial operation. In the radical resection group (group 3), one patient developed local recurrence and two patients had liver metastases within 1 year postoperatively. The other four patients underwent low anterior resection for synchronous rectal adenocarcinoma (7.0\%), and all were disease free with median follow-up of 2 years.
Subsequently, in overall six patients died of disease and their demographics were summarized in Table 3. The most common site of distant metastases was liver (100\%), followed by bone (33\%), lung (17\%), and pancreas (17\%). The overall 5-year disease-free survival was 87.0\%. Concerning different treatment methods, the 5year overall survival was $100 \%$ in patients following snaring polypectomy, $83 \%$ for those with local resection, and $63 \%$ for those with radical resection $(p=0.04)$ (Figure 1 ), whereas the 5 -year disease-free survival was $100 \%$ in patients following snaring polypectomy, $73 \%$ for those with local resection, and $51 \%$ for those with radical resection $(p=0.07)$ (Figure 2). Considering association of tumor size and prognosis, patients with tumor size of more than $10 \mathrm{~mm}$ showed significantly worse 5 -year diseasefree survival than those with less than $10 \mathrm{~mm}$ (37\% vs 97.1\%) $(p=0.001)$ (Figure 3).

\section{Discussion}

Rectal carcinoids are usually small at the initial presentation, and most studies reported a median size of $6 \mathrm{~mm}$, compared with a median size of $1-3 \mathrm{~cm}$ in colonic carcinoids [5,9]. Similarly, the median size of the rectal carcinoids in this study was $5 \mathrm{~mm}$, and it ranged from 1 to $60 \mathrm{~mm}$. Patients with rectal carcinoids usually present with local symptoms. Many were diagnosed incidentally during endoscopy (31.5\%). Carcinoid syndrome is associated with excessive secretion of serotonin, tachykinins, prostaglandins, catecholamines, and histamine. 5-Hydroxyindoleacetic acid (5-HIAA), their metabolite, was excreted in the urine, allowing it to be used as a diagnostic and postoperative surveillance marker. However, as hindgut carcinoid rarely secretes serotonin, carcinoid syndrome is uncommon and urinary 5-HIAA is seldom elevated [10]. This explains why in our study, only one patient had carcinoid syndrome and elevated urinary 5-HIAA (1/54). Immunohistochemical staining as an aid for the diagnosis of rectal carcinoid had also been explored in our study. Chromogranin A had a much lower sensitivity of $75.6 \%$ in our study, compared with 97.9\% for synaptophysin. Eriksson et al. suggested using plasma chromogranin A for monitoring of disease progression in midgut neuroendocrine tumors (NETs) especially in patients with metastatic disease, but not in hindgut NETs, since there was limited evidence to suggest similar applicability [11].

Table 2 Indications for further intervention after snaring polypectomy

\begin{tabular}{lllllll}
\hline & Inadequate margins & Large size & Abnormal imaging & Submuscosal lesion & Concomitant tumor & Total \\
\hline Snaring polypectomy only & N/A & N/A & N/A & N/A & N/A & $18(33.3 \%)$ \\
Transanal resection & $4(7.4 \%)$ & $4(7.4 \%)$ & $1(1.9 \%)$ & $1(1.9 \%)$ & $0(0 \%)$ & $10(18.5 \%)$ \\
TEO & $8(14.8 \%)$ & $5(9.3 \%)$ & $0(0 \%)$ & $2(3.7 \%)$ & $0(0 \%)$ & $15(27.8 \%)$ \\
Radical resection & $0(0 \%)$ & $3(5.6 \%)$ & $0(0 \%)$ & $0(0 \%)$ & $4(7.4 \%)$ & $7(13.0 \%)$ \\
\hline
\end{tabular}

N/A not applicable. 
Table 3 Data on patients' demographics who developed distant metastases

\begin{tabular}{llllll}
\hline Age & Sex & Size $(\mathbf{m m})$ & Treatment & Site of metastasis & Survival (months) \\
\hline 48 & M & 34 & LAR & Liver & 22 \\
53 & M & 25 & Not treated & Liver & 8 \\
64 & M & 60 & Not treated & Liver, bone & 1 \\
65 & F & 15 & Transanal excision, chemotherapy after metastasis & Liver, bone & 24 \\
74 & M & 25 & Transanal excision & Liver, pancreas & 13 \\
77 & F & 15 & LAR & Liver & 5 \\
\hline
\end{tabular}

Snaring polypectomy and endoscopic mucosal resection is an adequate treatment for most rectal carcinoids with small size [5,6]. In our study, one third of patients were diagnosed incidentally after endoscopic polypectomy, and no further intervention was needed. None subsequently developed local recurrence during follow-up. For larger tumors or those with margin involvement, local excision (either transanal excision or TEO) was performed. The latter approach could be employed for upper rectal lesions with better visualization and adequate margin with low morbidity [8]. However, both surgical approaches cannot remove potentially involved regional lymph nodes, which have been reported to have an incidence of up to $8.3 \%$ [7]. In our series, these patients had good outcome with a 5-year overall survival of $83 \%$ and 5 -year disease-free survival of $72 \%$, reflecting the adequacy of local treatment. From the limited number of patients who underwent local excision, we found that the use of TEO could achieve complete excision with clear margins. No patients had recurrence after the procedure. For large rectal carcinoids which required radical resection, the 5-year overall survival rate was $63 \%$ and disease-free survival was $50 \%$. Radical resection for this group of patients was necessary due to larger tumor size and the possibility of loco-regional lymph node metastasis [7]. The study showed that the 5-year overall survival was $100 \%$ in patients following snaring polypectomy, $83 \%$ for those with local resection, and $63 \%$ for those with radical resection, which is comparable with the reported 5-year survival rate of about $88 \%$ for all tumor stages $[5,12,13]$.

Size is an important and known prognostic factor for carcinoids, and those less than $10 \mathrm{~mm}$ are even considered benign $[5,6,12,13]$. Other important factors include the depth of invasion, lymphovascular invasion (LVI), and the number of mitotic figures [12,14-16]. Larger primary tumors in our study ( $>10 \mathrm{~mm}$ in size) were associated with a worse prognosis with 5-year survival of only $37 \%$, and this was consistent with previous reports $[2,5,12]$. Our metastatic rate was $9.3 \%$, consistent

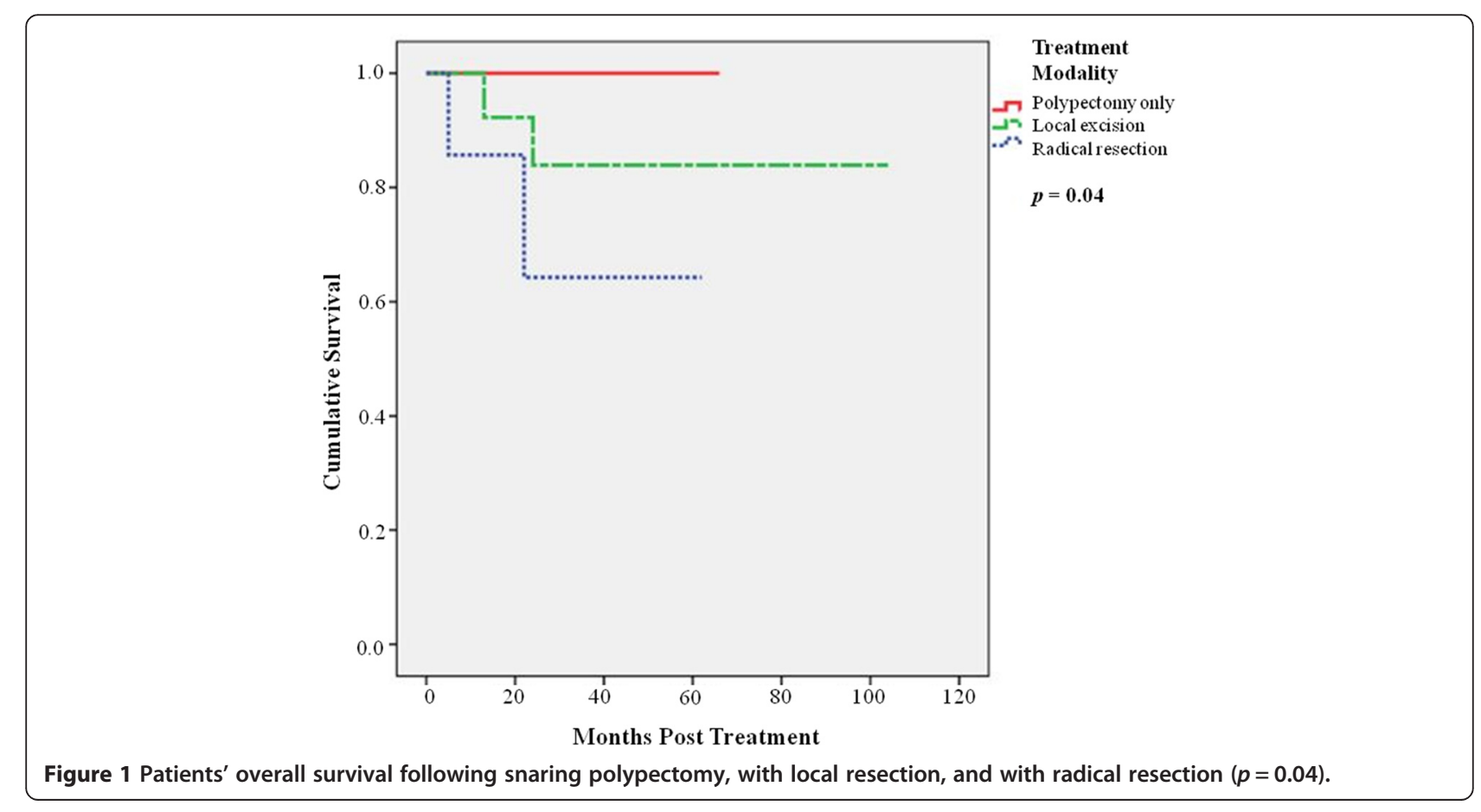




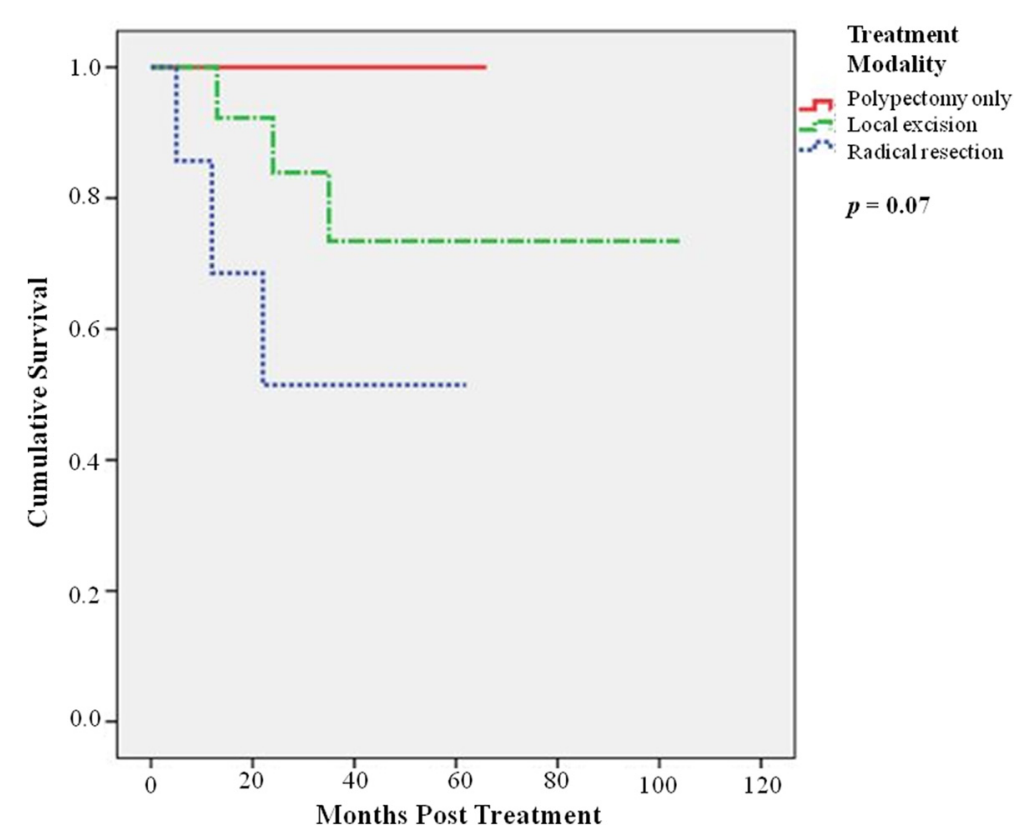

Figure 2 Patients' disease-free survival following snaring polypectomy, with local resection, and with radical resection $(p=0.07)$.

with other studies which ranged from $4 \%$ to $18 \%$ $[12,17,18]$. Mostly rectal carcinoids are localized with a low propensity of metastasis at the time of diagnosis, with the rate of metastasis ranging from $1.7 \%$ to $3.4 \%$ $[6,19]$. In the case of large tumors or perceived advanced disease, further imaging such as computed tomography, PET scans, or endorectal ultrasound is indicated $[5,7]$.
Synchronous non-neuroendocrine tumors were reported in the literatures with incidences of $25 \%$ to $46 \%$ [20,21]. Interestingly, most synchronous tumors were observed in the gastrointestinal tract, whereas metachronous tumors were more often observed outside the gastrointestinal tract $[13,20]$. As the presence of a metachronous malignancy was associated with more aggressive behavior

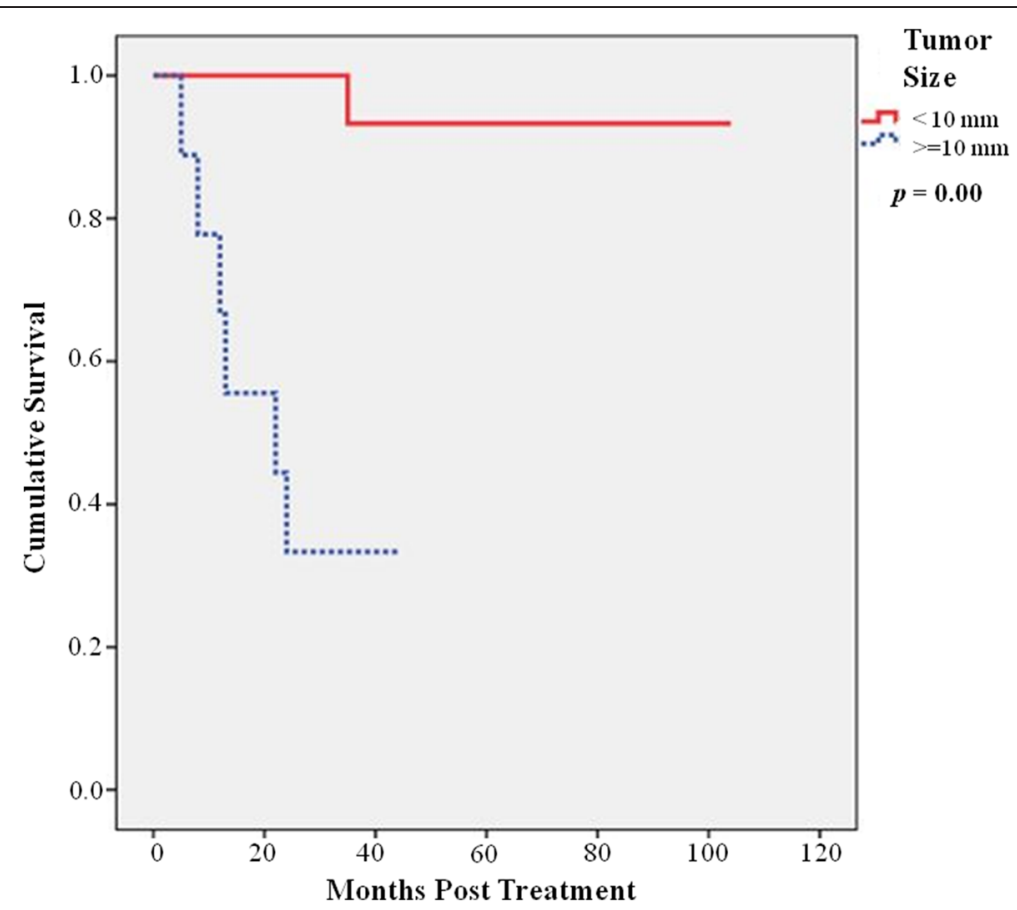

Figure 3 Patients with tumor size of more than $10 \mathrm{~mm}$ showed significantly worse 5 -year disease-free survival. 
in NET, close surveillance program was suggested in these cases [22]. In our study, four patients had synchronous carcinoma of rectum, and none of them developed metachronous tumors or recurrence.

Few clear recommendations are available on posttreatment surveillance of NETs $[5,7,23]$. In our center, the patients were followed up 2-3 monthly in the first two postoperative years, followed by lifelong annual assessment afterwards. Though surveillance endoscopy in this context had not been widely documented in medical literature, regular colonoscopy would be considered every $3-5$ years with an aim to rule out local recurrence and metachronous colorectal malignancy $[7,23]$. Although most local recurrences could be detected by digital examination and colonoscopy in our patients, some smaller submucosal recurrence could still be missed. Endorectal ultrasound in this case can play a role in identifying small submucosal recurrence, and it is recommended as a surveillance tool for monitoring local disease recurrence $[7,23]$. For those stage II/III diseases, surveillance with computed tomography would be essential to look for any distant metastases, particularly liver metastases, as we have been offering to our patients. As distant metastases may occur years after the initial treatment, long-term surveillance beyond 5 years had been suggested in patients with high-risk factors such as large size and advanced tumor staging with lymph node metastasis [23]. Chromogranin A is not commonly elevated in this group of patients, thus limiting their utilization [11].

\section{Conclusions}

Our data suggested that that local resection was an effective treatment for small rectal carcinoids and generally brought about good oncological and surgical outcomes. For larger tumors, radical resection seemed to provide acceptable oncological outcomes. Regular surveillance with colonoscopy and endorectal ultrasound is highly recommended for high-risk patients for long-term management. By sharing our experience, we hope to provide more evidence on the management on rectal carcinoids which, together with evidence from further studies, may guide us in the long-term management of these patients in the future.

\section{Competing interests}

The authors declare that they have no competing interests.

\section{Authors' contributions}

RW participated in the design of the study, performed the statistical analysis, and drafted the manuscript. OSH conceived the study, participated in the design of the study, and reviewed the draft of the manuscript. WL conceived of the study and helped in coordination and reviewed the draft of the manuscript. All authors read and approved the final manuscript.

\section{Acknowledgements}

We thank Dr. W.K. Shek for histolopathological support.

\section{References}

1. Habal N, Sims C, Bilchik AJ. Gastrointestinal carcinoid tumors and second primary malignancies. J Surg Oncol. 2000;75:310-6.

2. Konishi T, Watanabe T, Kishimoto J, Kotake K, Muto T, Nagawa H. Prognosis and risk factors of metastasis in colorectal carcinoids: results of a nationwide registry over 15 years. Gut. 2007:56:863-8.

3. Modlin IM, Lye KD, Kidd M. A 5-decade analysis of 13,715 carcinoid tumors. Cancer. 2003;97:934-59.

4. Modlin IM, Sandor A. An analysis of 8305 cases of carcinoid tumors. Cancer. 1997;79:813-29.

5. Yoon SN, Yu CS, Shin US, Kim CW, Lim SB, Kim JC. Clinicopathological characteristics of rectal carcinoids. Int J Colorectal Dis. 2010;25:1087-92.

6. Koura AN, Giacco GG, Curley SA, Skibber JM, Feig BW, Ellis LM. Carcinoid tumors of the rectum: effect of size, histopathology, and surgical treatment on metastasis free survival. Cancer. 1997;79:1294-8.

7. Holinga J, Khalid A, Fasanella K, Sanders M, Davison J, McGrath K. Metastatic risk of diminutive rectal carcinoid tumors: a need for surveillance rectal ultrasound? Gastrointest Endosc. 2012;75:913-6.

8. Maggard MA, O'Connell JB, Ko CY. Updated population-based review of carcinoid tumors. Ann Surg. 2004;240:117-22.

9. Landry CS, Brock G, Scoggins CR, McMasters KM, Martin 2nd RC. A proposed staging system for rectal carcinoid tumors based on an analysis of 4701 patients. Surgery. 2008;144:460-6.

10. de Herder WW. Biochemistry of neuroendocrine tumours. Best Pract Res Clin Endocrinol Metab. 2007;21:33-41.

11. Eriksson B, Oberg K, Stridsberg M. Tumor markers in neuroendocrine tumors. Digestion. 2000;62 Suppl 1:33-8.

12. Wang M, Peng J, Yang W, Chen W, Mo S, Cai S. Prognostic analysis for carcinoid tumours of the rectum: a single institutional analysis of 106 patients. Colorectal Dis. 2011;13:150-3.

13. Modlin IM, Kidd M, Latich I, Zikusoka MN, Shapiro MD. Current status of gastrointestinal carcinoids. Gastroenterology. 2005;128:1717-51.

14. Van Gompel JJ, Sippel RS, Warner TF, Chen H. Gastrointestinal carcinoid tumors: factors that predict outcome. World J Surg. 2004;28:387-92.

15. Soreide JA, van Heerden JA, Thompson GB, Schleck C, Istrup DM, Churchward M. Gastrointestinal carcinoid tumors: long-term prognosis for surgically treated patients. World J Surg. 2000:24:1431-6.

16. Mani S, Modlin IM, Ballantyne G, AhIman H, West B. Carcinoids of the rectum. J Am Coll Surg. 1994;179:231-48.

17. Zuetenhorst JM, Taal BG. Metastatic carcinoid tumors: a clinical review. Oncologist. 2005;10:123-31.

18. Shields $C J$, Tiret $E$, Winter DC. Carcinoid tumors of the rectum: a multi-institutional international collaboration. Ann Surg. 2010;252:750-5.

19. Fahy BN, Tang $L H$, Klimstra D, Wong WD, Guillem JG, Paty PB, et al. Carcinoid of the rectum risk stratification (CaRRs): a strategy for preoperative outcome assessment. Ann Surg Oncol. 2007;14:1735-43.

20. Gerstle JT, Kauffman Jr GL, Koltun WA. The incidence, management, and outcome of patients with gastrointestinal carcinoids and second primary malignancies. J Am Coll Surg. 1995;180:427-32.

21. Waisberg DR, Fava AS, Martins LC, Matos LL, Franco MI, Waisberg J. Colonic carcinoid tumors: a clinicopathologic study of 23 patients from a single institution. Arq Gastroenterol. 2009:46:288-93.

22. Federspiel BH, Burke AP, Sobin LH, Shekitka KM. Rectal and colonic carcinoids. A clinicopathologic study of 84 cases. Cancer. 1990;65:135-40.

23. Anthony LB, Strosberg JR, Klimstra DS, Maples WJ, O'Dorisio TM, Warner RR, et al. The NANETS consensus guidelines for the diagnosis and management of gastrointestinal neuroendocrine tumors (nets): well-differentiated nets of the distal colon and rectum. Pancreas. 2010;39:767-74. 